\title{
Intracranial Penetration During Temporal Soft Tissue Filler Injection-Is It Possible?
}

\author{
Wolfgang G. Philipp-Dormston, MD, ${ }^{*}$ Lara Bieler, MSc, ${ }^{\dagger \ddagger}$ Mathias Hessenberger,$\S$ \\ Thilo L. Schenck, MD, PhD, "Konstantin Frank, " Johann Fierlbeck, " \\ and Sebastian Cotofana, MD, PhD\#
}

\begin{abstract}
BACKGROUND Treating temporal volume loss for aesthetic and reconstructive purposes can be achieved by superficial or deep injections of soft tissue fillers into the temples. The latter is performed with bone contact that can lead to intracranial penetration when the bone is accidentally penetrated.
\end{abstract}

OBJECTIVE Based on a clinical case, the potential risk of accidental intracranial penetration was investigated.

MATERIALS AND METHODS Twenty fresh-frozen hemi-faces (all Caucasian ethnicity, 10 women, 10 men, mean age $72.8 \pm 11.2$ years) were investigated. Shape of pterion and bone-stability parameters of the temporal fossa were investigated. Bone stability was tested using uniaxial mechanical indentation (18-G, 1.25-mm diameter, 15- $\mathrm{mm}$ length blunt-tip device) until intracranial perforation occurred.

RESULTS Variations in the shape of the pterion, bone thickness, and density correlates were detected, however, without statistical significant differences in side symmetry. Minimum force necessary to penetrate intracranially was $40.4 \mathrm{~N}$. Maximum force generated by an 18-g, 70-mm length blunt-tip cannula was $32.1 \pm 4.2 \mathrm{~N}$ in $70 \mathrm{~mm}$ length and $75.3 \pm 10.2 \mathrm{~N}$ in $15 \mathrm{~mm}$ length.

CONCLUSION Based on the results of this investigation, it can be concluded that there is a risk for intracranial penetration performing the deep temple injection technique with direct pressure on the bone.

The authors have indicated no significant interest with commercial supporters.

$\mathrm{N}$ onsurgical approaches to reduce the signs of facial aging using soft tissue fillers are a steadily growing area of interest for dermatologic and other aesthetic practitioners. ${ }^{1}$ The increasing demand and popularity of these new therapeutic options has led to an increase in the application of soft tissue fillers between 2000 and 2016 by $298 \%$. $^{2}$ At the present time, 24 different soft tissue fillers have been approved by the U.S. Food and Drug Administration for various facial applications. ${ }^{3}$
Treatment options for the temporal region include the restoration of temporal volume loss, which can result from skin laxity, temporalis muscle atrophy, ${ }^{4}$ and/or from atrophy of the superficial and deep temporal fat pads. ${ }^{5}$ This presents clinically with a more prominent appearance of the temporal crest, the lateral superior orbital ridge, and the zygomatic arch.,

Techniques for treating temporal volume loss are primarily based on the injection of soft tissue fillers

\footnotetext{
*Cologne Dermatology, Hautzentrum Koeln, Klinik Links vom Rhein, Cologne, Germany; ${ }^{\dagger}$ Institute of Experimental Neuroregeneration, Paracelsus Medical University Salzburg and Nuremberg, Salzburg, Austria; ${ }^{*}$ Spinal Cord Injury and Tissue Regeneration Center Salzburg, Paracelsus Medical University Salzburg, Austria; ${ }^{\S}$ Institute of Anatomy, Paracelsus Medical University Salzburg and Nuremberg, Salzburg, Austria; "Department of Hand, Plastic and Aesthetic Surgery, Ludwig-Maximilians University, Munich, Germany; IIInstitute for Clinical Innovation, Paracelsus Medical University Salzburg and Nuremberg, Salzburg, Austria; "Department of Medical Education, Albany Medical College, Albany, New York
} 
into 2 different planes: superficial—into the subcutaneous plane, superficial to the superficial temporal artery and the motor branches of the facial nerve-and deep-into the temporalis muscle, in contact with the bone and deep to the motor branches of the facial nerve and deep to the middle zygomatico-temporal vein (sentinel vein). ${ }^{8-10}$ However, severe complications have been reported when treating the temple of which irreversible blindness is considered the worst of any potential outcome. ${ }^{11-13}$ The true causes for this complication remain elusive, but explanatory models include intravascular injection of the material with ultimate occlusion of the retinal artery. ${ }^{11}$ To avoid this potential risk, deep supra-periosteal injections, in contact with the bone, were recommended. ${ }^{11,14}$

The underlying bone in this area is known as the pterion and has been shown to vary in shape as it is a point of convergence of the sutures between the frontal, sphenoid, parietal, and squamous temporal bones. ${ }^{15-}$ ${ }^{18}$ This variability of bone morphology can result in a reduction of bone stability giving rise to the potential risk of intracranial penetration during deep temporal augmentation procedures.

Inspired by a clinical case of potential intracranial penetration that occurred after a temporal augmentation procedure, the objectives of the present study were the following:

(1) To describe a clinical case where accidental intracranial penetration is suspected

(2) To measure bone stability parameter of the temporal fossa and

(3) To reproduce the clinical scenario (presented in the case report) using an 18-G, 70-mm blunt-tip cannula.

\section{Clinical Case}

Bilateral temporal augmentation of a 48-year-old healthy woman without previous neurological signs or symptoms was performed in an external clinic using a commercially available soft tissue filler (Juvederm Voluma with Lidocaine, Allergan, Dublin, Ireland). The box contained 2 syringes of the volumizing product, 2 single-use 23-G, 1" U.T.W. (Ultra Thin Wall) needles, and 2 single-use, 18-G, 70-mm cannulae. The injection was first performed on the right side using the deep injection technique: skin perforation of the temple was performed using the $23 \mathrm{G}, 1^{\prime \prime}$ needle at the temporal skin-hairline border approximately at the horizontal level of the lateral canthus. After the skin was penetrated, the needle was removed and the 18-G, 70-mm blunt-tip cannula was inserted into deeper layers. When being in contact with the bone, additional pressure was experienced by the patient followed by a sudden loss of resistance and a pain-free breakthrough sensation. When treating the contralateral side, a slight burning sensation started which intensified during the next 3 hours. Three hours after the injection, slight bruising and right-sided headache started. The following day, nausea, stiffness of the neck, severe headache, and complete bruising of the temporal region were present (Figure 1). No central or peripheral neurological deficiencies were experienced by the patient. Oral corticosteroids and analgesics were selfadministered, but no hyaluronidase was injected. Magnetic resonance imaging and computer tomographic imaging 8 days after the injection revealed no definite evidence for intracranial penetration, but local signs of dural inflammation in the area of the injection were diagnosed with no affection of the fronto-insular cortex or presence of any other pathology. Fourteen weeks after injection, general symptoms cleared almost completely and the follow-up magnetic resonance imaging (MRI) scan revealed local dural thickening; this was interpreted as suspect of a highly differentiated glioma (Figure 1). Eighteen weeks after injection, another follow-up MRI scan showed a hypointense, cortical signal alteration indicative of a previous local corticocerebral inflammation. No evidence for glioma in a C11 methionin positron emission tomography scan was noted 22 weeks after the injection procedure. The final radiological report 14 months after the injection described a hereditary local disregulation of the gray-white matter differentiation as the most probable cause when summarizing the imaging-based findings. Albeit accidental intracranial injection of soft tissue filler was considered as a valid explanatory model for the imaging-based findings, this was not concluded in the written record because of the lack of magnetic resonance reference data, the uniqueness of the case and because of the lack of experience with injectable related complications. Up to date, the patient never experienced any central or peripheral neurological deficiency, but still perceives intermittent 

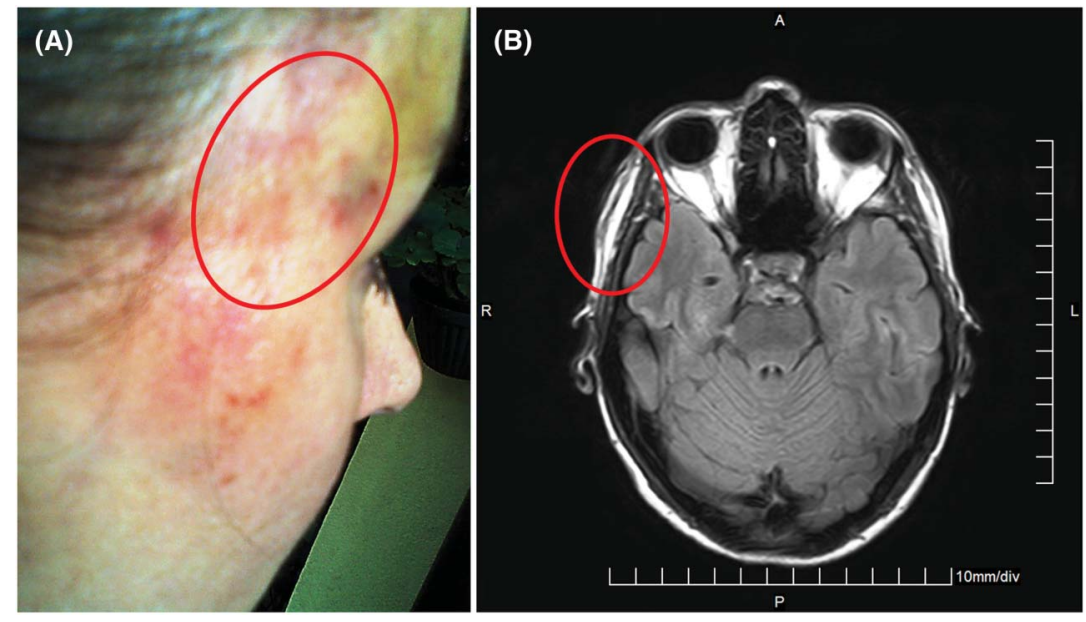

Figure 1. Clinical $(A)$ and magnetic resonance image $(B)$ of the presented case showing a hematoma of the right temporal region as well as increased thickness of the subcutaneous structures as compared to the contralateral side.

headaches and postural unilateral pain of the temporal region.

\section{Materials and Methods}

Based on the presented clinical case, a biomechanical investigation was conducted to simulate the clinical scenario using the same cannula size and length, to evaluate if intracranial penetration is possible in a testing environment, and to provide valid data for the possibility of intracranial penetration when using soft tissue fillers applying with the deep injection technique.

\section{Study Sample}

Twenty fresh-frozen, not embalmed hemi-faces of 10 Caucasian human body donors ( 5 women and 5 men) with a mean age at death of $72.8 \pm 11.2$ years (range 49-88) and a body mass index of $27.3 \pm 6.2 \mathrm{~kg} / \mathrm{m}^{2}$ (range 19.9-38.1) were investigated. None of the body donors was diagnosed while alive with plasmacytoma nor had a documented uptake of bisphosphonates. Two (1 man and 1 woman) were diagnosed while alive with osteoporosis, whereas 7 had a positive medical history for smoking. While alive, all body donors gave informed consent for their bodies to be used for medical education and for purposes of scientific investigation.

\section{Selection of Measured Locations}

Each of the hemi-faces was freed from soft tissues within the area of interest using sharp anatomical dissection. The shape of the pterion was identified and documented. Four different measuring points for mechanical testing were selected and marked on the bone in relation to the lateral canthus based on the most frequently used locations for temporal augmentation procedures: A1: $2 \mathrm{~cm}$ posterior and $2 \mathrm{~cm}$ superior to the lateral canthus, A2: $2.5 \mathrm{~cm}$ posterior and $2 \mathrm{~cm}$ superior to the lateral canthus, B1: $2 \mathrm{~cm}$ posterior and $1 \mathrm{~cm}$ superior to the lateral canthus, and B2: $2.5 \mathrm{~cm}$ posterior and $1 \mathrm{~cm}$ superior to the lateral canthus (Figure 2).

\section{Mechanical Testing}

Mechanical testing relied on uniaxial mechanical compression using a static material testing machine (Z5.0; Zwick/Roell, Ulm, Germany). Mechanical compression was performed perpendicular to the surface of the bone at the 4 marked measuring points (A1, A2, B1, B2) using an 18-G, 15-mm solid, blunt-tipped wire as an indentation device. Indentation was carried out until penetration of the bone and access to the intracranial space was achieved or until the maximal producible load of the testing machine (here 480 Newton $[\mathrm{N}]$ ) was exceeded.

Additionally, the maximal force generated by an 18 G, 70-mm blunt-tip cannula (INEX, PuiseuxLe-Hauberger, France) was measured (Figure 3) using the same uniaxial compression set-up. As the distance between the bone and the strong, 


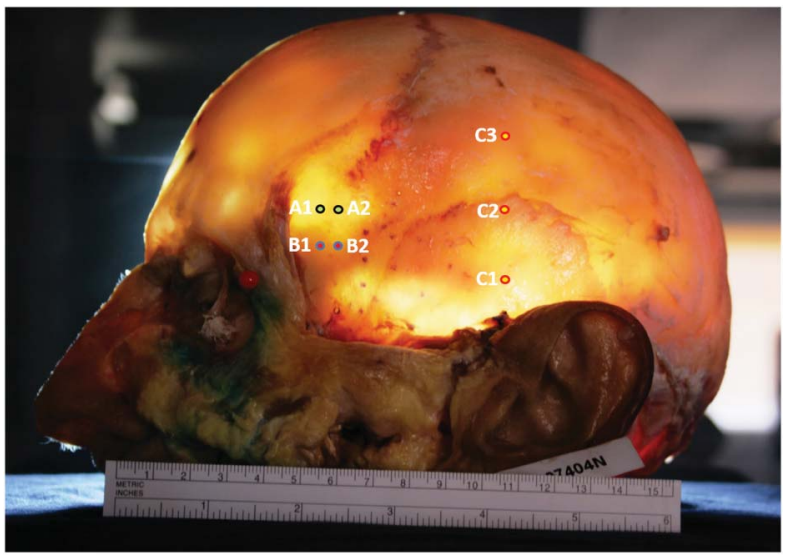

Figure 2. Image of a transilluminated cephalic specimen showing the 4 measuring points for uniaxial mechanical compression: $\mathrm{A} 1: 2 \times 2 \mathrm{~cm}, \mathrm{~A} 2: 2.5 \times 2 \mathrm{~cm}, \mathrm{~B} 1: 2 \times 1 \mathrm{~cm}$ and B2: $2.5 \times 1 \mathrm{~cm}$ in posterior $\mathrm{x}$ superior relation to the lateral canthus (red dot) at the level of the canthal plane. Also, the 3 additional points for translumination imaging using a monochromatic light source are included which were used for the pseudo-color gray-value analysis of the obtained images: $\mathrm{C} 1: 5 \times 0 \mathrm{~cm}$, C2: $5 \times 2 \mathrm{~cm}$ and C3: $5 \times 4 \mathrm{~cm}$ in posterior $x$ superior relation to the lateral canthus (red dot) at the level of the canthal plane.

fibrous deep temporal fascia was measured to be in mean $15 \mathrm{~mm}$ in the tested region, the cannula was cut to a size of $15 \mathrm{~mm}$ and the generated forces were measured for this length as well. Transcortical bone thickness was manually measured in each of the 4 mechanical testing locations after each indentation procedure.

\section{Translumination Analyses}

Bone density correlates of the hemisected cephalic specimens were measured from standardized pictures taken with a standard digital camera (EOS, 450D; Cannon, Tokyo, Japan) with fixed adjustment for exposure time, focus, sensitivity to light, and diaphragm during translumination. Pseudo-color grayvalue analysis of the obtained images was performed using Image J software (National Institutes of Health, Sacaton, AZ) with values ranging from 0 (black) to 1,000 (bright white). Pseudo-color gray-value analysis was performed at the 4 points used for mechanical testing and at 3 additional randomly selected points. These, too, were in relation to the lateral canthus: $\mathrm{C} 1$ : $5 \mathrm{~cm}$ posterior and $0 \mathrm{~cm}$ superior, $\mathrm{C} 2: 5 \mathrm{~cm}$ posterior and $2 \mathrm{~cm}$ superior, and C3: $5 \mathrm{~cm}$ posterior and $4 \mathrm{~cm}$ superior (Figure 2).

\section{Statistical Analyses}

To show the variability of the bones of the temporal region, the shape of the pterion was classified and bone density correlates measured at the 7 points were calculated and compared for sex and side differences using a student $t$-test. Bone thickness and the maximal force leading to intracranial penetration were measured and correlated using Pearson R correlation test. The maximal force generated by the $18-\mathrm{G}$ cannula in both the 70 -mm and 15 -mm lengths was measured
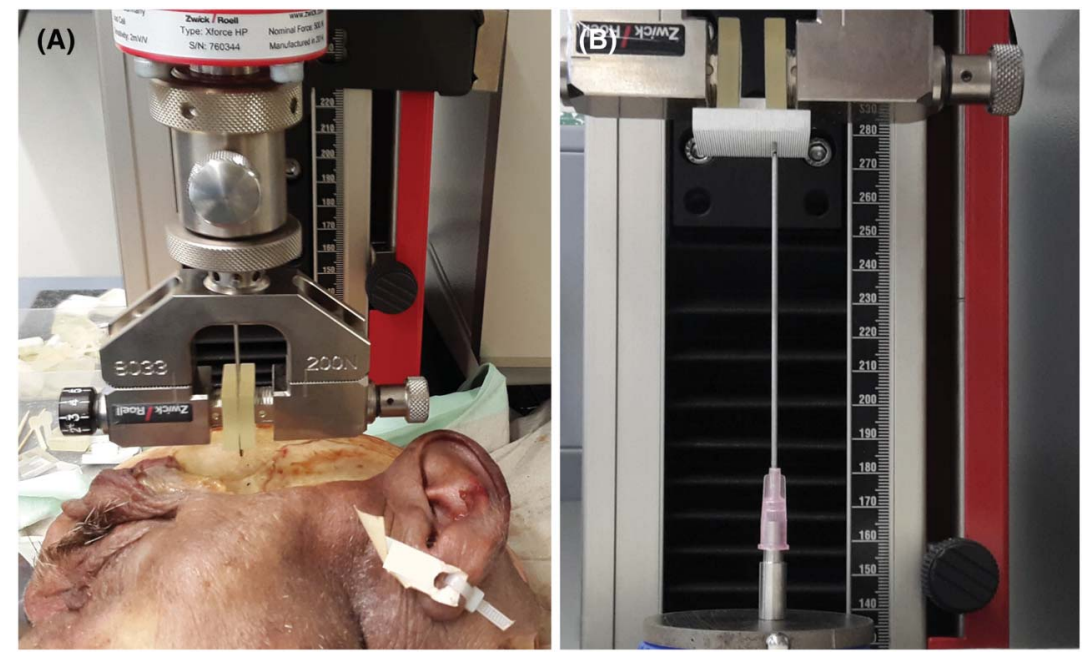

Figure 3. Panel A, showing the set-up of the indentation procedure using a solid, blunt wire (18 G [1.25 $\mathrm{mm}], 15 \mathrm{~mm}$ in length) as indentation device. Panel B, showing the uniaxial mechanical compression of the 18-G, 70-mm blunt cannula for measuring the maximal force generated on compression. 
and compared with the forces needed to penetrate intracranially (Figure 3). Statistical analyses were performed with SPSS Statistics 23 (IBM, Armonk, NY) and all applied tests were 2-sided, and $p$-values <.05 were considered as statistically significant to guide conclusions.

\section{Results}

The shape of the pterion of the 20 hemi-faces was sphenoparietal in 70\% $(n=14)$, fronto-temporal in $5 \%(n=1)$, and epipteric bones were present in $25 \%$ $(n=5)$. No significant differences were detected between sex $(p=.75)$ or side $(p=.75)$.

Bone density (as measured by pseudo-gray value analysis) was $156 \pm 10.9$ units for men and 96.4 \pm 6.9 units for women (mean \pm SD) with a mean sex difference of $59.5 \pm 23.1$ units $(p=.004)$. Side differences were without statistical significance $(p=.55)$ with a mean value of $10.2 \pm 19.8$ units.

Overall bone thickness (independent of measured location) was $4.52 \pm 2.01 \mathrm{~mm}$ (range: $1.70-13.0 \mathrm{~mm}$ ) with women having a thicker bone $(5.60 \pm 2.25 \mathrm{~mm})$ as compared to men $3.43 \pm 0.92 \mathrm{~mm}(p=.01)$. No significant side differences were detected for overall thickness measures $(p=.85)$ nor at each of the 4 (A1, A2, B1, B2) individually measured points $(p>.29)$.

Incomplete intracranial penetration resulted in 6 cases ( 3 women) as the maximal force capacity of the testing machine $(480 \mathrm{~N})$ was exceeded because of increased bone resistance independent of the indentation sites (A1, A2, B1, B2). Overall, mean penetration force (independent of indentation site) was $294 \pm 130 \mathrm{~N}$ (range: 40-480), whereas after excluding the incomplete penetrated cases $(n=6)$ the mean force overall was $216 \pm 53 \mathrm{~N}$. The minimum force to penetrate intracranially was $40.4 \mathrm{~N}$ (Figure 4) which was measured $2.5 \mathrm{~cm}$ posterior and $1 \mathrm{~cm}$ superior to the lateral canthus; (B2). A ranking of the locations according to the minimal force needed for intracranial penetration revealed the following: $\mathrm{B} 2<$ $\mathrm{A} 1<\mathrm{B} 1<\mathrm{A} 2(2.5 \times 1 \mathrm{~cm}, 2 \times 2 \mathrm{~cm}, 2 \times 1 \mathrm{~cm}, 2.5 \times 2 \mathrm{~cm}$; all posterior $\times$ superior as measured from the lateral canthus; Table 1).

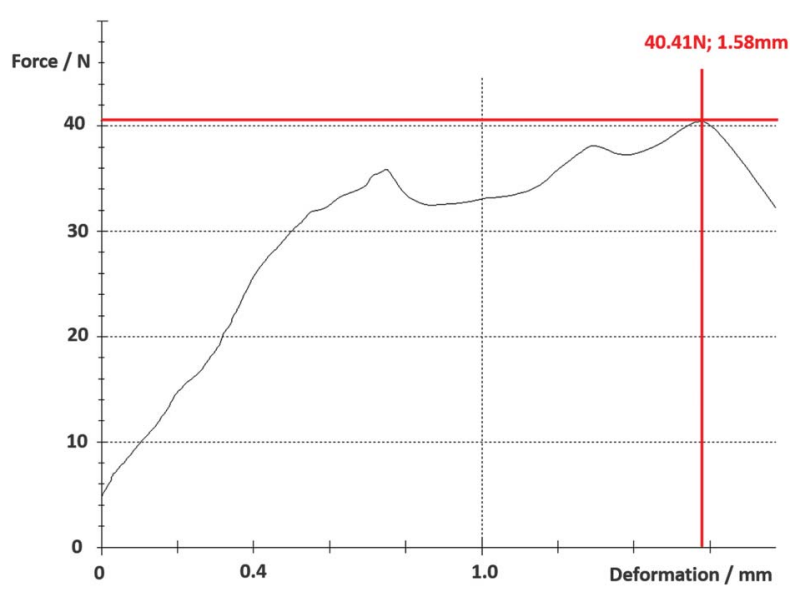

Figure 4. Force (measured in Newton (N)/deformation (measured in $\mathrm{mm}$ ) diagram of the lowest measured force needed for intracranial penetration, obtained at $2.5 \times 1 \mathrm{~cm}$ (posterior $\times$ superior) to the lateral canthus (B2): $40.4 \mathrm{~N}$. Wavy shape of the curve displays increased forces during the penetration of the outer and inner cortical lamella of the diploe of the respective bone.

A statistically significant correlation was identified between bone thickness and applied force until intracranial penetration was achieved $\mathrm{r}=0.56(p=.01)$, but not between bone density and applied force until intracranial penetration.

The mean maximal force that was generated by the 18 G cannula was $32.1 \pm 4.2 \mathrm{~N}$ (range 27.0-38.6) in its full length $(70 \mathrm{~mm})$, whereas at $15 \mathrm{~mm}$ in length, the mean maximal force was $75.3 \pm 10.2 \mathrm{~N}$ (range 61.385.9). These values were independent of the speed during uniaxial mechanical compression $(5,20,50$ $\mathrm{mm} / \mathrm{s}$; data not shown).

\section{Discussion}

The objectives of this study were inspired by the events of a clinical case which occurred after a temporal augmentation procedure in which the volumizing material was injected using the deep application technique, i.e., being in contact with the bone. The authors tested the bone stability of the temporal fossa in selected locations using an indentation device with identical dimensions to those of the cannula used in the presented clinical case (18-G, 70-mm blunt-tip). The authors also tested the maximal force than can be generated by the cannula used in the clinical case to reveal whether intracranial penetration in this setting is possible. 
TABLE 1. Measured Data on Bone Thickness, Bone Density (As Measured by Pseudo-Color Gray-Value Analyses), and Bone Stability (i.e., the Force in Newton Needed to Penetrate Intracranially) of the 20 Investigated Hemi-Faces

\begin{tabular}{|c|c|c|c|c|c|c|}
\hline \multirow[b]{2}{*}{$n=20$} & \multicolumn{2}{|c|}{ Bone Thickness $/ \mathrm{mm}$} & \multicolumn{2}{|c|}{ Bone Density/Units } & \multicolumn{2}{|c|}{ Bone Stability/N } \\
\hline & Mean (SD) & Range & Mean (SD) & Range & Mean (SD) & Range \\
\hline A1 & $3.72(2.5)^{*}$ & $1.9-13.0$ & $136(61)^{*}$ & $40-255$ & $295(132)^{*}$ & $126-480$ \\
\hline $\mathrm{A} 2$ & $4.70(2.4)^{*}$ & $1.7-12.0$ & $121(61)^{*}$ & $45-257$ & $302(137)^{*}$ & $110-480$ \\
\hline B1 & $4.79(1.8)$ & $2.5-9.0$ & $115(67)^{*}$ & $11-264$ & $300(135)^{*}$ & $108-480$ \\
\hline B2 & $4.86(2.5)^{*}$ & $2.3-12.5$ & $106(64)^{*}$ & $10-285$ & $279(162)^{*}$ & $40-480$ \\
\hline Overall & $4.51(2.0)^{*}$ & $1.7-13.0$ & $126(44)^{*}$ & $10-285$ & $294(130)^{*}$ & $40-480$ \\
\hline
\end{tabular}

The pterion is a point of convergence of the sutures between the frontal, sphenoid, parietal, and squamous temporal bones. ${ }^{15}$ Deep to this bony landmark, the anterior branch of the middle meningeal artery, ${ }^{19}$ Broca motor speech area (Brodmann area 44, 45), ${ }^{17}$ the Sylvian point (division of the stem of lateral sulcus of the cerebral hemisphere into the anterior, ascending, and posterior limb), ${ }^{18}$ and the insula all can be found intracranially deep to the pterion (Figure 5). Bone thickness in this area varies between $1 \mathrm{~mm}$ and $5 \mathrm{~mm}$ and differences between sex and side have been previously reported. ${ }^{17,19-21}$ The thickness measurements are in line with these previous reports and the novel concept to measure bone density by pseudocolor gray-value analysis supported the data as we did find variations between sex and side (albeit statistically not meaningful). It is of great clinical importance, however, as each temporal region should be treated carefully and differences in side or sex must be considered when treating each individual.

Previous studies have described cutaneous access points for temporal augmentation procedures and relied on cutaneous landmarks, such as the tail of the eyebrow or the temporal hair line, ${ }^{22}$ whereas others emphasized guidance based upon "one up, one over" in relation to the temporal crest and the superior orbital rim when promoting safe and effective injection locations. ${ }^{9}$ As cutaneous landmarks change with age- because of skin laxity and changes in the hairline-or with other cosmetic procedures (change in length or position of the eyebrow) or because of variation in the angulation of the needle/cannula when penetrating the skin and deeper structures, ${ }^{9}$ it might be
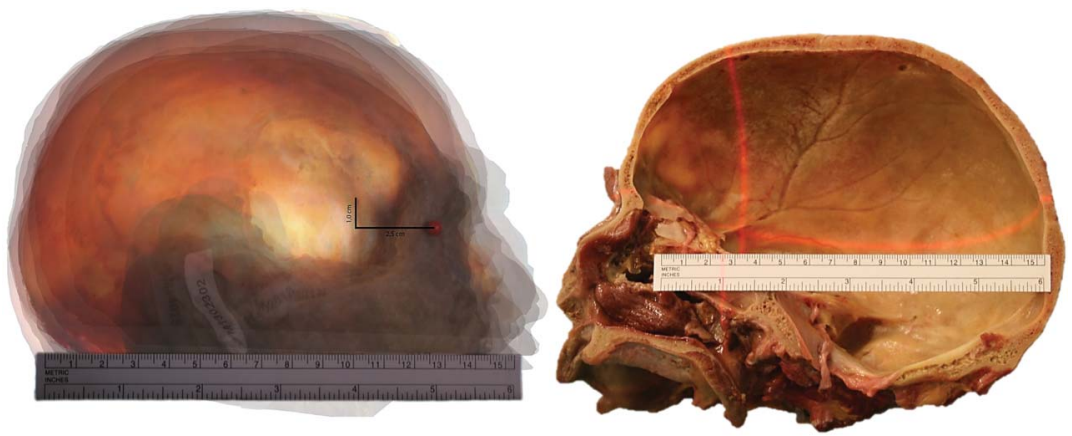

Figure 5. Left panel: external view of the 10 superimposed right cephalic specimens during transillumination using a unifocal light source, showing dense and less dense areas of the temporal region. The point of minimal bone resistance (as measured in this investigation) was $2.5 \times 1 \mathrm{~cm}$ (posterior $\times$ superior) to the lateral canthus (red mark, B2): $40.4 \mathrm{~N}$. Right panel: internal view of a right cephalic specimen showing the same location as indicated by the laser cross. Note the bony impression of the course of the middle meningeal artery. 
potentially safer to rely on stable and easy-to-identify landmarks to guide temporal augmentation procedures. In the present investigation, the measurements relied on such an easy-to-identify and stable bony landmark-the lateral canthus in the horizontal plane at the level of the pupil—and provided information on bone stability in selected locations posterior ( 2 or 2.5 $\mathrm{cm}$ ) and superior ( 1 or $2 \mathrm{~cm}$ ) to the lateral canthus. The location with the lowest bone stability was measured to be $2.5 \mathrm{~cm}$ posterior and $1 \mathrm{~cm}$ superior to the lateral canthus (Figure 5); here, $40.4 \mathrm{~N}$ was sufficient to penetrate intracranially which is equivalent to a pressurization of 4.12 kilogram. During temporal augmentation procedures, this point should be marked and direct bone contact in this location, independent of where the superficial structures were penetrated, should be avoided.

In the experimental setting, the maximal force generated by the $18-\mathrm{G}, 70-\mathrm{mm}$ blunt-tip cannula was $38.6 \mathrm{~N}$ and was lower when compared with the smallest force needed to penetrate intracranially $(40.4 \mathrm{~N})$. However, the investigated sample size relied only on 10 human body donors and it can be suspected that with increasing sample size the variation of minimal bone stability increases and cases with a bone stability of less than $40.4 \mathrm{~N}$ could be encountered. It also has to be stated that the sample investigated was of Caucasian ethnicity exclusively, had a mean age of $72.8 \pm 11.2$ years (range 49-88) and had a positive tabacco history in $70 \%$ of the cases, which potentially deviates from the patients seen for temporal augmentation procedures seen on a daily clinical basis.

In the testing scenario, we reduced the size of the cannula to $15 \mathrm{~mm}$ in length and could measure a maximal generated force of $85.9 \mathrm{~N}$ before deformation of the cannula occurred. Increased cannula stability can be further enhanced by the surrounding structures of the puncturing canal, such as the temporalis muscle, the strong fibrous deep temporal fascia, and the superficial temporal fascia. Such an increase in cannula stability (up to $85.9 \mathrm{~N}$ ) and forces greater than the minimal bone stability $(40.4 \mathrm{~N})$ can lead to an increased risk of intracranial penetration. These increased forces, however, can be achieved only when the cannula is stable and placed perpendicular to the bone surface. It should be however noted, that the presented forces were generated in a testing scenario and might not re-create the actual anatomical forces at play when injecting a live patient. Nevertheless, it is recommended to approach the bone from a different location than the skin perforation using angulation to avoid a direct perpendicular approach. Doing so, will reduce the direct forces onto the bone and can reduce the risk of this potentially life-threatening complication. A location superior to the inferior temporal septum seems to be a favorable one for skin penetration and access to deeper structures as no motor branches of the facial nerve nor the superficial temporal artery are identified here. ${ }^{5,8,9}$

The indentation device used in this study as well as the selected cannulae for measuring the mechanical properties was both blunt and thick in diameter (1.25 $\mathrm{mm})$. It can be suspected that using sharp devices or devices with a smaller diameter, the applied forces can surpass the bony stability of the temporal fossa easily and the risk for intracranial penetration is higher than in the tested scenarios. However, future studies need to test these assumptions, which potentially might lead to the development of pressure sensitive devices for the application of soft tissue filler for the temporal region when treating temporal volume loss.

From the clinical perspective, this study tries to create awareness for the potential risk of accidental intracranial penetration. The performed experiments were enrolled in a very specific setting and potentially do not reflect the injection procedures performed on a daily routine. Albeit, very specific in its results, this study also reveals that practitioners should be able to differentiate between symptoms related to the injection procedure itself or extracranial adverse events, such as soreness, jaw pain, and headache and symptoms related to intracranial penetration, such as nausea, vomiting, and neck stiffness.

\section{Conclusion}

Based on the results of these analyses, it can be concluded that there is a potential risk of intracranial penetration when the deep injection technique is 
performed being in perpendicular orientation to the bone surface. The measured variability in the shape of the pterion as well as the differences in bone stability between side and sex underline this risk. The synopsis of the biomechanical measurements and the imagingbased findings presented in the case report increase the probability that intracranial penetration without major neurological damage occurred in the clinical case presented. Profound knowledge of the temporal anatomy as well as the use of a gentle injection technique allowing periosteum contact, but no direct pressure on the bone should be mandatory for physicians treating this delicate region.

\section{Acknowledgments The authors thank Andreas}

Traweger and Herbert Tempfer from the Institute of Tendon \& Bone Regeneration, Paracelsus Medical University Salzburg \& Nuremberg, Salzburg, Austria for their tremendous support during the biomechanical testing procedures.

\section{References}

1. Chacon AH. Fillers in dermatology: from past to present. Cutis 2015; 96:E17-9.

2. Surgeons ASoP. Cosmetic Plastic Surgery Statistics. 2016. Available from: https://www.plasticsurgery.org/documents/News/Statistics/2015/ plastic-surgery-statistics-full-report-2015.pdf. Published 2016. Accessed July 1, 2016

3. Services USDoHaH. Soft Tissue Fillers Approved by the Center for Devices and Radiological Health. U.S. Food and Drug Administration; 2017. Available from: https://www.fda.gov/MedicalDevices/ ProductsandMedicalProcedures/CosmeticDevices/WrinkleFillers/ ucm227749.htm. Accessed July 1, 2016.

4. Juhasz ML, Marmur ES. Temporal fossa defects: techniques for injecting hyaluronic acid filler and complications after hyaluronic acid filler injection. J Cosmet Dermatol 2015;14:254-9.

5. O'Brien JX, Ashton MW, Rozen WM, Ross R, et al. New perspectives on the surgical anatomy and nomenclature of the temporal region: literature review and dissection study. Plast Reconstr Surg 2013;132: $461 \mathrm{e}-3 \mathrm{e}$.

6. Friedman O. Changes associated with the aging face. Facial Plast Surg Clin North Am 2005;13:371-80.
7. Coleman SR, Grover R. The anatomy of the aging face: volume loss and changes in 3-dimensional topography. Aesthet Surg J 2006;26:S4-9.

8. Cotofana S, Schenck TL, Trevidic P, Sykes J, et al. Midface: clinical anatomy and regional approaches with injectable fillers. Plast Reconstr Surg 2015;136:219S-34S.

9. Sykes JM, Cotofana S, Trevidic P, Solish N, et al. Upper face: clinical anatomy and regional approaches with injectable fillers. Plast Reconstr Surg 2015;136:204S-18S.

10. Sykes JM. Applied anatomy of the temporal region and forehead for injectable fillers. J Drugs Dermatol 2009;8:s24-7.

11. Beleznay K, Carruthers JD, Humphrey S, Jones D. Avoiding and treating blindness from fillers: a review of the world literature. Dermatol Surg 2015;41:1097-117.

12. Chen Y, Wang W, Li J, Yu Y, et al. Fundus artery occlusion caused by cosmetic facial injections. Chin Med J (Engl) 2014;127:1434-7.

13. Lazzeri D, Agostini T, Figus M, Nardi M, et al. Blindness following cosmetic injections of the face. Plast Reconstr Surg 2012;129:995-1012.

14. Jung W, Youn KH, Won SY, Park JT, et al. Clinical implications of the middle temporal vein with regard to temporal fossa augmentation. Dermatol Surg 2014;40:618-23.

15. Adejuwon SA, Olopade FE, Bolaji M. Study of the location and morphology of the pterion in adult nigerian skulls. ISRN Anat 2013 2013:403937.

16. Saxena SK, Jain SP, Chowdhary DS. A comparative study of pterion formation and its variations in the skulls of Nigerians and Indians. Anthropol Anz 1988;46:75-82.

17. Oguz O, Sanli SG, Bozkir MG, Soames RW. The pterion in Turkish male skulls. Surg Radiol Anat 2004;26:220-4.

18. Khatri Chirag R, Gupta D, Soni D. Study of pterion and incidence of epipteric bones in dry human skulls of Gujarat. NJIRM 2012;3:57-60.

19. Ma S, Baillie LJ, Stringer MD. Reappraising the surface anatomy of the pterion and its relationship to the middle meningeal artery. Clin Anat $2012 ; 25: 330-9$

20. Apinhasmit W, Chompoopong S, Chaisuksunt V, Thiraphatthanavong $\mathrm{P}$, et al. Anatomical consideration of pterion and its related references in Thai dry skulls for pterional surgical approach. J Med Assoc Thai 2011;94:205-14.

21. Hwang K, Kim JH, Baik SH. The thickness of the skull in Korean adults. J Craniofac Surg 1999;10:395-9.

22. Breithaupt AD, Jones DH, Braz A, Weinkle S. Anatomical basis for safe and effective volumization of the temple. Dermatol Surg 2015;41(Suppl 1):S278-83.

Address correspondence and reprint requests to: Sebastian Cotofana, MD, PhD, Department of Medical Education, Albany Medical College, 47 New Scotland Avenue MC135, Albany, NY 12208, or e-mail: cotofas@mail.amc.edu 